\title{
Study of Dynamic Solid-Liquid Interfacial Phenomena in Hypereutectic Al-Si Alloy Using in situ HRTEM.
}

\author{
Matthew M. Schneider ${ }^{1}$, James M. Howe ${ }^{1}$ \\ 1. Department of Materials Science and Engineering, University of Virginia, Charlottesville, VA, USA.
}

Solid-liquid interfaces are extensively encountered in materials processing and applications. The structure and properties of these interfaces affect solidification, wetting behavior, and crystalline growth mechanisms, including epitaxial growth. The exact structural and chemical nature of these interfaces has been historically difficult to study at high resolution due to the fact that one phase is a liquid. Recently, in situ HRTEM experimental methods have progressed to enable the study of solid-liquid interfaces in metals with resolution on the atomic-scale [1-4]. Reported results thus far include direct observation of step/ledge growth [1], various crystallographic facets present during growth/dissolution[1,2], ordering of liquid layers adjacent to crystalline solids [1-4], and chemical segregation at interfaces [2,5]. This work reports first observations of new interfacial phenomena associated with the dynamic behavior of a highindex solid-liquid facet at equilibrium.

Spray-atomized nanoparticles of AA390 alloy (Valimet, Inc.) ranging in size from 100 to $300 \mathrm{~nm}$ were used in the present investigation. The particles were dispersed by ultrasonication in ethanol. TEM grids coated with $<5 \mathrm{~nm}$ of amorphous carbon (Ted Pella Inc., Ultrathin Type A) were used as supports for the nanoparticles. The particles are enclosed by a native oxide layer approximately $4 \mathrm{~nm}$ thick; this contained the molten phase during observation at high temperature.

An FEI Titan 80-300 TEM operated at $300 \mathrm{kV}$ was used for high-resolution observation of interfaces of interest. Samples were melted in situ with a double-tilt heating holder (Gatan 652-Ta). In an effort to eliminate thermal drift issues caused by temperature variation across the sample holder, the entire system was thermally equilibrated at $650^{\circ} \mathrm{C}$ for one hour before observation. In order to control temperature changes within a given nanoparticle without inducing thermal drift, the electron beam itself was used to control temperature changes by spreading/condensing it on the region of interest. Screen capture software was used to record videos from the Gatan Multiscan 794 camera output at a temporal resolution of two frames per second. Due to limitations in temporal resolution, study was restricted to interfaces close to their equilibrium state. Both EELS (Gatan Tridiem) and EDS (EDAX Titan 300 ST) were employed to study the chemical distributions near the interfaces of interest.

The most commonly observed facets on the primary Si particles were $\{111\}$ and $\{113\}$, as previously reported [2]. The $\{113\}$ facet is the fastest growing of the two and as growth progresses, the Si particles are ultimately bounded by $\{111\}$ facets [1]. The solid-liquid interface along the $\{113\}$ plane consists of single atom steps alternating between the $\{111\}$ and $\{002\}$ planes, as shown in Figure 1 . When inclined slightly, as in Figure 2, the $\{111\}$ plane terminations on this interface were observed to have a corrugated structure with planes jutting out one or two atomic distances into the liquid.

The dynamic motion of the ends of several $\{111\}$ planes along the $\{113\}$ interface were tracked over 5 seconds at a temporal resolution of 2 frames per second. The interface position in the images was outlined by connecting the terminus of each $\{111\}$ plane along the interface. An unsharp mask was applied to aid in the accurate and consistent identification of each plane terminus. This processing 
helped reduce the high-spatial frequency noise present in the images and to enhance the contrast of the features present above this threshold, as evident in Figures 2A and 2B. Care was taken to ensure that the identification of the interface location was unaffected by this process; i.e., images were compared plane-by-plane, and frame-by-frame both before and after the unsharp masking process to ensure accuracy. The interface positions from subsequent video frames were offset horizontally and combined to allow clear interpretation of variation from frame-to-frame, as shown in Figure $2 \mathrm{C}$. Additionally, a light gray line was added as a stationary reference plane to Figure $2 \mathrm{C}$.

The average position of the interface remained unchanged across the 10 frames show in Figure $2 \mathrm{C}$, in spite of the large number of fluctuations that occurred along the interface. The atomic structure of the $\{113\}$ interface leads to atomic troughs parallel to the [110] viewing direction, as shown Figure 1. One side of the atomic trough is composed of the terminating $\{111\}$ planes in contact with the liquid. The series of interface profiles in Figure $2 \mathrm{C}$ shows that the amplitude of the solid-liquid interface fluctuations is no more than one or two atomic distances, and that these fluctuations occur with a periodicity of one or two trough-distances along the interface. Previous work has shown similar behavior for an incoherent solid-solid interface in a massive phase transformation [6]; to our knowledge, this is the first report of this type of dynamic interfacial behavior at a relatively high-index solid-liquid interface.
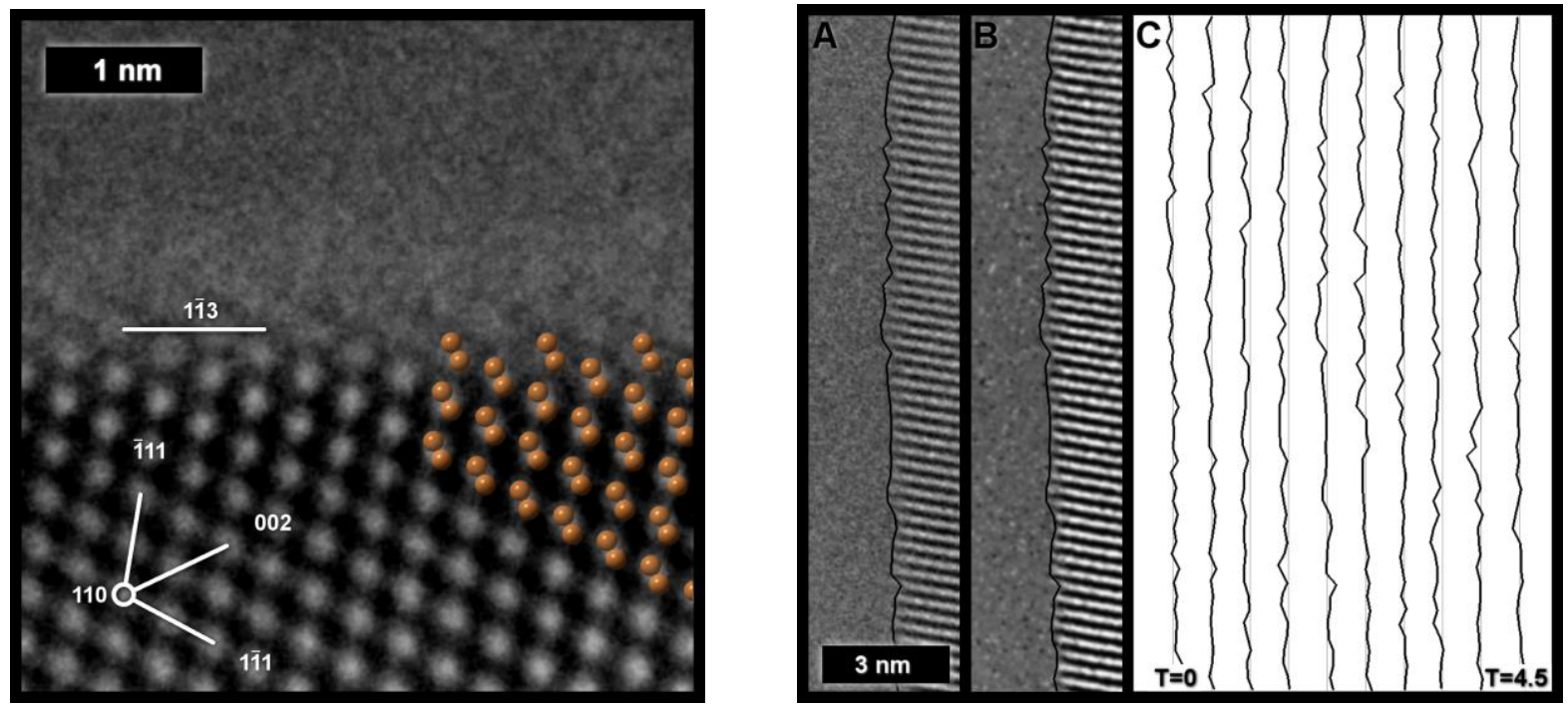

Figure 1 - HRTEM image of interface between crystalline Si and liquid Al-rich alloy. An atomic model is overlaid on the right-hand side to illustrate the structure of the $\mathrm{Si}$ at the $\{113\}$ interface.

Figure 2 - Tracking termination of $\{111\}$ planes by outlining their terminus on the image (A), an unsharp masked image (B), and the interface location outlined across ten frames and offset horizontally (C) with the gray line inserted to reference the average position.

\section{References:}

[1] S Arai et al, Journal of Electron Microscopy 48(4) (1999), p. 317-321.

[2] JM Howe, H Saka, MRS Bulletin 29(12) (2004), p. 951-957.

[3] SH Oh, C Scheu, M Rühle, Korean Journal of Electron Microscopy 1 (2006), p.

19-24. [4] SH Oh et al, Science 310(5748) (2005), pg. 661-663.

[5] SK Eswaramoorthy, JM Howe, and G Muralidharan, Science 318(5855), p. 1437-1440. [6] N

Raffler, JM Howe, Metallurgical and Materials Transactions A 37A (2006) p. 873-878. 